\title{
Cutaneous manifestations in COVID 19 patient: A case report
}

\author{
Elena Barbagelata, Elena Bernero, Roberta Garbarini and Marco Scudeletti \\ Internal Medicine Department, General Lavagna Hospital, Genoa, Italy
}

\begin{abstract}
Coronavirus disease 2019 (COVID-19), caused by severe acute respiratory syndrome coronavirus 2 (SARS-CoV-2), is a current, ongoing life-threatening crisis and it has become a global public health emergency. The early diagnosis and management of the disease remains a major challenge.

Although most patients with COVID-19 manifest fever and respiratory tract symptoms, SARS-CoV-2 infection may also involve other organs/systems and present with extra-respiratory manifestations. Occasionally, these extra-respiratory clinical expressions represent the initial presentation of SARS-CoV-2 infection, prior to fever or respiratory manifestations.

Cutaneous manifestations of COVID-19 disease have not yet been fully described. Some skin disorders in COVID-19 might result in missed diagnosis. An important question is whether the dermatological problem in COVID-19 has any clinical impact on diagnosis and treatment of disease. We describe this unusual case report of the extra-respiratory manifestations of COVID-19 in order to help clinicians to better understand the range of clinical presentations associated with SARS-CoV-2 infection, allowing appropriate management of this disease.
\end{abstract}

\section{Introduction}

The outbreak of COVID-19 has been declared a Public Health Emergency of International Concern by the World Health Organization (WHO) and presents a great challenge for the health care communities across the globe $[1,2]$. Syndrome coronavirus 2 [SARS-CoV-2] has been diagnosed in more than 2.4 million people worldwide [3].

SARS-CoV 2 is an enveloped virus composed of positive sense single-stranded RNA and belongs to the coronavirus family. The virus enters cells through the angiotensin converting enzyme 2 (ACE2) receptor, found on the surface of cells [4]. The lungs are the primary site of infection for COVID-19, with patients presenting symptoms ranging from a mild flu-like symptoms to fulminant pneumonia and potentially lethal respiratory distress. Common clinical features of this virus infection inside and outside of the respiratory system include fever, cough, headache, diarrhea, fatigue, headache, and myalgia [5]. With the exponential increase of infected patients worldwide, clinical characteristics of COVID-19 are being better defined and new symptoms are emerging.

Dermatological symptoms are reported sporadically. In a recent review on clinical characteristics of coronavirus disease 2019 in China, skin manifestations were observed in $0,2 \%$ of cases [6].

Recently, Recalcati and colleagues reported the cutaneous manifestations of COVID-19 infection in Italy and asked for more papers to confirm and better understand the pattern of skin involvement with COVID-19 cases reporting cutaneous manifestations [7].

The purpose of this paper is to report an emblematic case of a patient with COVID-19 with atypical clinical presentation and associated skin manifestations .

\section{The case}

On May 2020, a 40-year-old man was admitted in Emergency department because of about a month fever and dry cough. Remote medical history was of allergic diathesis (apricots, pollen), no weight loss, no smoker, recent dental surgery.

Because of respiratory symptoms, in the suspicion of SARSCOV2 infection he started home therapy with hydroxychloroquine (HCQ). First PCR SARSCOV2 research performed by oropharyngeal swab was negative and HCQ was suspended after three tablets, subsequently supplemented with amoxicillin/clavulanate for persistence of fever.

10 days before admission in hospital, painful inguinal swelled lesions appeared, for which he performed ambulatory venous ultrasound for deep vein thrombosis or lymphoadenopathy resulted negative, a subcutaneous painful nodule was present in the right groin.

When he was admitted at hospital he was eupnoic, he had fever $\left(39^{\circ} \mathrm{C}\right)$ and symmetrical nodular skin lesions in the arms, followed by appearance of a diffuse papular macular rash. Thoraco-abdominal objectivity was negative. No neurological signs.

Laboratory tests showed elevated inflammation indices (ESR, ferritin, PCR) with marked neutrophilic leukocytosis and lymphopenia, elevation of the alpha1 and alpha2 component at protein foresis,

${ }^{\star}$ Correspondence to: Elena Barbagelata, department of internal Medicine, Lavagna General Hospital, Via don Bobbio 43, 16033, Genoa, Italy, E-mail: elenabarbagelata@yahoo.it

Key words: COVID-19, SARS-CoV-2, Cutaneous manifestations

Received: January 08, 2021; Accepted: January 13, 2021; Published: January 15, 2021 
negative procalcitonin, increase of transaminases and cytolysis indices, increase in BNP values.

Initial presentation was as sepsis with gnarled erythema. Differential diagnosis included a systemic (autoimmune or drug-based) vasculitis, inflammatory bowel disease, paraneoplastic syndrome (mainly AML or MDS), viral infection.

Piperacillin tazobactam therapy (18 g / day) and diagnostic path were started.

Negative blood cultures and urine culture, ANA, ENA and ANCA immunology, serology for borreliosis and other viral and bacterial infections (CMV, EBV, HIV, HBV, HCV, TOXO test, weil felix), nasopharyngeal swab and quantitative serology for SARS COV2. Complement and ACE test were normal. No pleuroparenchymal lesions at chest radiography. Troponine, BNP and transaminases were high and systolic function was at the lower limits of the norm (FE 50\%) at echocardio. Respiratory exchanges maintained (Sat O2 =95\%).

Pharyngeal swab resulted positive for actinomyces odontolyticus.

48 hours later, we observed extensive confluence of maculopapular lesions such as diffuse erythema (Figure 1), reappearance of nodular lesions on the arms bilaterally in the context of which vesicles and bullous lesions containing citrine liquid on the shoulders bilaterally developed (Figure 1). Clinical worsening occured with high fever $\left(39.8^{\circ} \mathrm{C}\right)$, cough, asthenia. We performed total body CT and skin biopsy.

Piperacillin tazobactam was replaced with levofloxacin ( $1 \mathrm{~g} / \mathrm{die})$ on suspicion of adverse reaction and high-dose steroid therapy was started (methylprednisolone $2 \mathrm{mg} / \mathrm{Kg} /$ die).

TC showed the presence of modest left lung subpleural infiltrates (Figure 2).

Two days after treatment change, defervescence and resolution of lesions on the skin occured. Echocardio improvement in systolic function (FE 56\%); troponine, BNP and transaminases normalization.

Skin vesicles swab was negative for SARSCOV2.

On histological examination "flap of skin with normotrophic and orthokeratotic epidermis with multiple vesicles full of inflammatory elements, mainly neutrophilic granulocytes, in an abscess-like form; in the "dispersed" inflammatory infiltrated dermis represented by lymphocytes, neutrophilic granulocytes, eosinophilic granulocytes and histiocytes “.

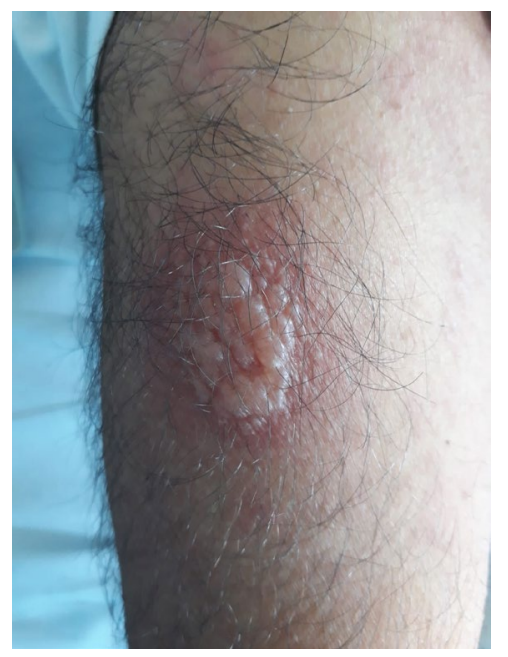

Figure 1. Extensive confluence of maculopapular lesions such as diffuse erythema

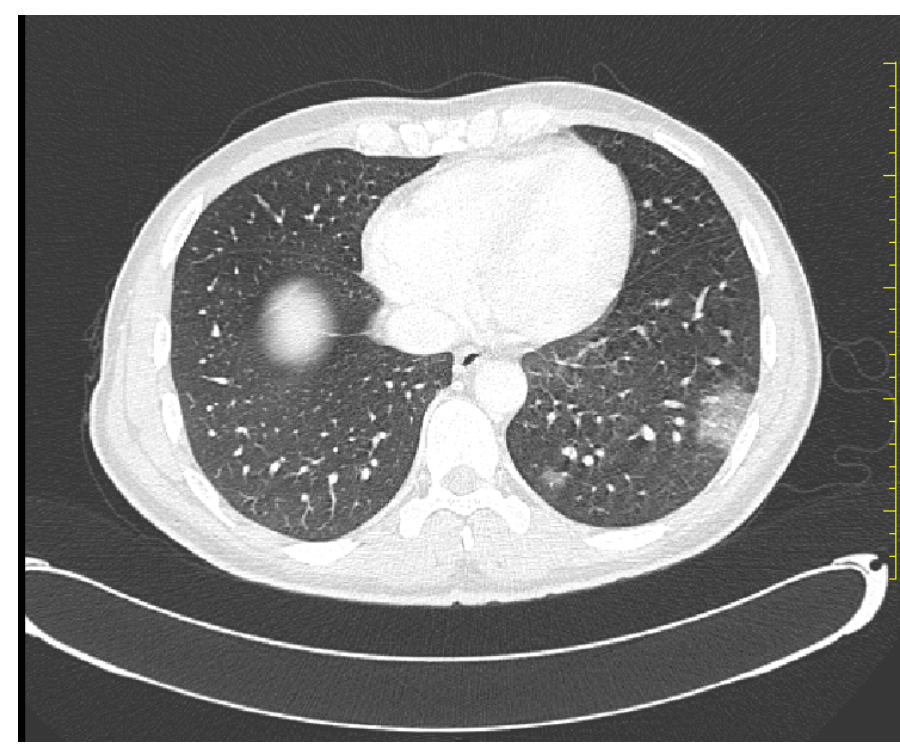

Figure 2. TC showed the presence of modest left lung subpleural infiltrate

Table 1. Revised diagnostic criteria of Sweet Syndrome

\section{Major criteria}

1. Abrupt onset of tender or painful erythematous plaques or nodules, occasionally with vesicles, pustules, or blisters

2. Predominantly neutrophilic dermal infiltrate without leukocytoclastic vasculitis Minor criteria

1. Preceded by a nonspecific respiratory or gastrointestinal tract infection or vaccination or associated with:

- Inflammatory diseases such as chronic autoimmune disorders, infections

- Hemoproliferative disorders or solid malignant tumors

- Pregnancy

2. Fever $>38^{\circ} \mathrm{C}$

3. Abnormal laboratory values at presentation (three of four):

- Erythrocyte sedimentation rate $>20 \mathrm{~mm} / \mathrm{h}$

- Elevated C-reactive protein levels

- Leukocytosis $>8,000$

- Neutrophilia $>70 \%$

4. Excellent response to treatment with systemic corticosteroids or potassium iodide

The intense infiltration of neutrophilic granulocytes led to suspicion of diffuse dermatitis Sweet's type. The good response to steroid therapy also supports clinical suspicion (Table 1).

\section{Discussion}

Dermatological manifestations of COVID 19 are reported sporadically.

In Zhao, et al. systematic review, skin manifestations occurred on average 9.92 days after the onset of systemic symptoms; moreover, the longest incubation of lesions was 30 days, and about $15 \%$ of patients had skin lesions as the first symptom The most common skin lesion reported was erythema and distributed on patients' trunk, extremities, flexural regions, face and mucous membranes [8].

The pathogenesis of cutaneous manifestations in COVID- 19 remains unknown. Skin rash may be directly induced by virus infection as is common in other viral diseases, for example, dengue, rubella, and measles. Hyperactive immune response, complement activation and microvascular injury have been implicated.

Sweet's syndrome is characterized by high fever, diffuse erythematous nodules (nodulo-bullous at the hands), leukocytosis with absolute neutrophilia. 
At lung, neutrophilic alveolitis characterized by interstitial infiltrate and nodules is described, it presents with cough and dyspnea. Often associated with infections, mainly of the upper respiratory tract, lymphoproliferative diseases, solid neoplasms, drugs (Granulocyte colony-stimulating factor, Sulfamethoxazole + Trimethoprim, Bortezomib, azathioprine, hydrossichloroquine). Etiology is related to a cytokine-mediated hypersensitivity reaction followed by neutrophilic infiltration [9].

At a subsequent check, our patient was positive for PCR search of SARSCOV2.

Indeed, SARSCOV2 infection is associated with heterogeneous skin manifestations. Preliminary data describe cases of maculopapular skin rash (Grover's disease type), morbilliform exanthems of the trunk complicated in rash, papulovesicular eruptions [10].

Skin involvement appears be related to lymph-mediated vasculitis, stimulated by the presence of viral particles in the small vessels. The inflammatory reaction with vasodilation, spongiosis also with activation of Langherans cells would cause keratinocytes lesions and would therefore be the cause of heterogeneous clinical manifestations. It would be an immune-mediated mechanism of aberrant response by the host. To confirm this, similar findings were recognized in other tissues of patients affected by COVID, such as in the lung where diffuse alveolar damage, lymphocyte and neutrophil interstitial infiltrates and hyaline membrane formation [11] had been found.

Another theory identifies disseminated intravasal coagulation in small venous vessels and the consequent phenomena of microthrombosis as a triggering event.

It is not currently clear whether the skin manifestations are secondary to the respiratory infection or primary site of infection, it could be another interesting research topic, we know that our patient PCR search for virus on the vesicular serum was negative.

Raffaele Gianotti provided histopathological evidence by biopsy, finding viral particles in the cutaneous blood vessels in patients with COVID-19 infection [12]. Recently, Recalcati raised a hypothesis that peculiar lesions that were unreported in previous literature may represent late clinical manifestations of COVID-19 infection among young healthy individuals [7]. To clarify the underlying mechanisms, large-scale prospective studies with biopsies, serological tests showing antibody response to virus infection and PCR analysis of suspected patients are warranted. Since the time kinetics of skin exanthem and viremia probably vary among different infections, it may be significant to measure blood viral load at different time points (before, during, or after lesions onset), thereby determine the appropriate time of biopsies for molecular identification.

\section{Conclusion}

Understanding how COVID-19 present on the skin may help health practitioners to recognize and manage the disease. More clinical data should be collected, and more researches should be carried out for a better understanding of the cutaneous manifestations caused by COVID-19.

\section{References}

1. Coronavirus Disease (COVID-19) - Events as They Happen [Internet]. Rolling Updates on Coronavirus Disease (COVID-19)., World Health Organization, 2020.

2. Han Y, Yang H (2020) The transmission and diagnosis of 2019 novel coronavirus infection disease (COVID-19): a Chinese perspective. J Med Virol 92: 639-644. [Crossref]

3. World Health Organization, Coronavirus Disease (COVID-19) Outbreak Situation [Internet] [cited 2020 Apr 22]. Available from:, World Health Organization, 2020

4. Guo YR, Cao QD, Hong ZS, Tan YY, Chen SD, et al. (2020) The origin, transmission and clinical therapies on coronavirus disease 2019 (COVID-19) outbreak-an update on the status. Mil Med Res 7: 1. [Crossref]

5. Henry D, Ackerman M, Sancelme E, Finon A, Esteve E (2020) Urticarial eruption in COVID-19 infection. J Eur Acad Dermatol Venereol 34: e244-e245. [Crossref]

6. Guan WJ, Ni ZY, Hu Y, Liang WH, Ou CQ, et al. (2020) Clinical Characteristics of Coronavirus Disease 2019 in China. N Engl J Med 382: 1708-1720. [Crossref]

7. Recalcati S (2020) Cutaneous manifestations in COVID-19: a first perspective. $J$ Eur Acad Dermatol Venereol 34: e212-e213. [Crossref]

8. Zhao Q, Fang X, Pang Z, Zhang B, Liu H, et al. (2020) COVID-19 and cutaneous manifestations: a systematic review. J Eur Acad Dermatol Venereol 34: 2505-2510. [Crossref]

9. Nofal A, Abdelmaksoud A, Amer H, Nofal E, Yosef A, et al. (2017) Sweet's syndrome: diagnostic criteria revisited. J Dtsch Dermatol Ges 15: 1081-1088. [Crossref]

10. Sachdeva M, Gianotti R, Shah M, Lucia B, Tosi D, et al. (2020) Cutaneous manifestations of COVID-19: Report of three cases and a review of literature. $J$ Dermatol Sci 98: 75-81. [Crossref]

11. Xu Z, Shi L, Wang Y, Zhang J, Huang L, et al. (2020) Pathological findings of COVID-19 associated with acute respiratory distress syndrome. Lancet Respir Med 8: 420-422. [Crossref]

12. Gianotti R, Zerbi P, Dodiuk-Gad R (2020) Histopathological study of skin dermatoses in patients affected by COVID-19 infection in the Northern part of Italy. J Cosmet Dermatol Sci Appl 98: 141-143. [Crossref]

Copyright: (C2021 Barbagelata E. This is an open-access article distributed under the terms of the Creative Commons Attribution License, which permits unrestricted use, distribution, and reproduction in any medium, provided the original author and source are credited. 